\title{
Advanced Noise Abatement Departure Procedures: Custom Optimized Departure Profiles
}

\author{
Sander J. Hebly* \\ National Aerospace Laboratory, 1059 CM Amsterdam, The Netherlands \\ Hendrikus G. Visser ${ }^{\dagger}$ \\ Delft University of Technology, 2629 HS Delft, The Netherlands
}

\begin{abstract}
This paper presents the concept of custom optimized departure profiles, as an advanced form of noise abatement departure procedures. This concept relies on fixed routes in combination with individually optimized vertical departure profiles. Although some environmental performance is lost by fixing the ground track, an increase in complexity associated with free routing is eliminated as well, leading to a concept that does not seem to be incompatible with today's Air Traffic Control principles. By using a primitive form of trajectory negotiation between airline and ATC, selected flights can be allowed to perform an optimized departure without interfering with non-participating traffic. Apart from the concept itself, this paper also describes the departure profile optimization tool, which is based on a previously developed trajectory optimization tool called NOISHHH. Finally -in a numerical example- a current standard ICAO-A procedure is compared with two optimized profiles for a Boeing 737 departure from Amsterdam Airport. Fuel burn, noise impact and the required time to a specified point are compared for the three departure profiles.
\end{abstract}

\section{Nomenclature}

$=$ number of awakenings

$=$ drag

$=$ specific energy

$=$ awakenings multiplication factor

$=$ distance-based coordinate

$=$ thrust

$=$ velocity

$=\mathrm{x}$-direction coordinate

$=\mathrm{y}$-direction coordinate

$=\mathrm{z}$-direction coordinate

$=$ path angle

$=$ thrust setting

$=$ roll angle

$=$ fuel flow

$=$ heading

\section{Introduction}

$\mathrm{T}$

The noise resulting from flight operations at major airports is a continuing source of annoyance in nearby residential communities. This being recognized by the industry, new aircraft generations continue to be less noisy than their predecessors, but this development in itself does not solve the noise problem in a fast growing market. Therefore a range of operational measures has been implemented at airports located close to sensitive communities.

\footnotetext{
${ }^{*}$ Ph.D. Candidate, Environment and Policy Support, sheblij@nlr.nl, Member AIAA.

${ }^{\dagger}$ Associate Professor, Faculty of Aerospace Engineering, h.g.visser@tudelft.nl, Associate Fellow AIAA. 
A very common operational measure is the use of noise abatement procedures ${ }^{1}$. For example, two well known types of noise abatement departure procedures (NADP) are the so-called distant and close-in NADPs. Procedures that belong to one of these types are designed to bring noise relief either close to or somewhat more distant from the airport. Departure procedures like this have been in use for decades now. Less widely used are noise abatement arrival procedures, with the (advanced) continuous decent approach (CDA) as a well known example. Although the details with respect to the CDA may differ from location to location, the general idea is that aircraft remain higher and use less engine power during the approach, especially somewhat further away from the airport ${ }^{2}$. Apart from a noise reduction, this also offers an opportunity for fuel savings, making it attractive not only to the residential communities, but also to the aircraft operators.

A characteristic that is shared among all these type of procedures is that they are generic in nature, i.e. they are not optimized with respect to the local situation. This means that although they have been optimized for a particular shift in noise load, they are not designed based on noise impact, as this is very much dependent on the population distribution around the airport. Among previous research projects that do take the actual local situation into consideration are several trajectory optimization studies, in which the trajectory optimization is applied to a specific arrival or departure ${ }^{3-5}$. Such studies have been performed for arrivals as well as for departures and the results with respect to noise exposure reduction are impressive. Research in this area is still being performed and refined and similar new projects in this direction have been initiated as well recently ${ }^{6}$.

Although these fully optimized trajectories can offer substantial benefits with respect to their environmental impact, several problems arise with respect to a potential implementation. For example, the proposed trajectories are definitely more complex from the pilot's point of view, and not directly compatible with today's navigation and guidance principles. A second problem that can be indentified is a considerable increase in airspace complexity ${ }^{7}$. With each aircraft flying its own optimized trajectory, the regular traffic patterns as known today will no longer be present. This not only leads to a far more complex situation for air traffic controllers, the situational awareness of the pilots can be impacted as well, especially with respect to nearby traffic.

When assuming that the navigation and guidance difficulties can be overcome by near future levels of flight deck automation, the airspace complexity remains the dominating problem. This paper presents a concept that does not suffer from the increase in complexity, but is still able to significantly reduce environmental impact by means of trajectory optimization. This concept is based on fixed (published) Area Navigation (RNAV) routes, combined with individually optimized or so to say custom made departure profiles for each flight.

The details of the proposed concept are first explained in section II, including the responsibilities of the different parties involved. The next two sections of this paper present the departure profile optimization tool and a corresponding implementation strategy. Section $\mathrm{V}$ presents a selection of numerical results, followed by the concluding remarks in section VI.

\section{Conceptual Framework}

Looking at the current situation based on RNAV SIDs, all aircraft fly similar ground tracks. However, their actual profiles can differ substantially. Even under similar atmospheric conditions, numerous causes can be identified that lead to these differences in the vertical plane. Examples include performance differences between different types of aircraft, aircraft instantaneous weight (payload and fuel), company procedures and training, and possibly even crew habits, etc.

The concept of optimized departure profiles, when compared to trajectories optimized in all dimensions, shows a very clear advantage with respect to airspace complexity. Adding flights that fly optimized custom profiles does not necessarily lead to greater variety in departure profiles for the different flights. At the same times it also does not automatically result in a more complex traffic situation for air traffic controllers. For them it could even increase the predictability of aircraft behavior with respect to speed and altitude, if somehow provided with the appropriate information.

Basically, it is the similarity in resulting trajectories of both the current situation and the proposed concept that prevent the increase in air space complexity. It is the very same principle that leads to a very important second benefit: its suitability for mixed concepts or mixed operations. Both using traditional procedures as well as optimized profiles simultaneously in the same terminal area seems possible, without the need for segregation of both traffic streams. With respect to a potential implementation, this property would make it less complicated to perform trials and it also allows for a gradual transition, or possibly a partial one, as will be discussed later in this section.

Evidently, not optimizing with respect to the horizontal plane reduces the benefits that can be expected from trajectory optimization. The performance penalty in terms of fuel burn and noise reduction that arises is simply the price to be paid for a reduced complexity. However, the proposed concept does not rule out all lateral optimization. 
Optimal routes can still be computed, as long as there is a single acceptable result for all traffic destined for a specific SID and the published departure routes are updated accordingly. The best method of determining the ideal fit, which will probably be some kind of weighted average, is currently under investigation, as is the magnitude of the resulting performance penalty.

The next three subsections describe which parties would be involved and what their responsibilities would be in the concept of optimized custom departures profiles, starting from the airline perspective. These sections together also discuss why it would be worthwhile for an airline to invest in such a system.

\section{A. Airline responsibility}

Although the actual profile optimization and selection for a specific flight could be made the responsibility of the Air Navigation Service Providers (ANSPs), the optimal departure profiles concept assumes that it remains with the airlines, typically as a task of the Airline Operations Centers (AOCs) or Operations and Control Centers (OOCs). There are several reasons for this task allocation, which will be discussed in this section.

Realistic trajectory optimization or even simulation requires detailed and accurate knowledge with respect to the aircraft. Aircraft weight for example is currently unknown to the ANSP, but known to the airline, especially at takeoff. Clearly, the aircraft weight status could easily be shared, but it can be a lot more complicated for other parameters. Airlines have very detailed information with respect to the performance of their aircraft, as provided by the manufacturers (e.g. Performance Engineers Manuals). As this data is considered proprietary, ANSPs normally have to use higher-level performance data, for example like EUROCONTROL's Base of Aircraft DAta (BADA). Therefore, it is expected that the airlines have access to more accurate and more reliable models.

Placing the profile optimization responsibility with the airlines also seems to fit the concept of the Business Trajectory (BT), an important element of the Single European Sky ATM Research Programme (SESAR) ${ }^{8}$. The BT is the trajectory that best represents the airspace users intentions for a specific flight. The airspace users own their BTs and are responsible for updating them if constraints (including those arising from infrastructural and environmental restrictions) would require so. Related to this principle and typical for the departure phase is the selection of some sort of reduced thrust mode for a specific departure (de-rate, flex, etc.). For the optimal departure profiles concept, it is still the airline that is free to perform the trade-off for this decision, just like it is in the current situation.

As previously mentioned, a partial implementation seems perfectly possibly at first sight, where airlines are free to decide on taking part in the optimized departure profiles mechanism. Airlines choosing not to join in can simply continue to fly the standard procedures, as they do today. Those who decide otherwise will have to commit to investments, required to realize the concept. In this decision, it is eventually all about the cost of the system and the benefits that can be expected from it. At the same time it is conceivable that airlines that do participate would not do so for all of their aircraft types. Some types could simply be more suitable for this concept than others, mostly dependent on the navigation capabilities of the aircraft.

There are several kinds of benefits that can be expected from flying optimized profiles. First, there are opportunities for costs savings. For example, the possibility to fly fuel optimal trajectories can be exploited in order to realize fuel savings. And with carbon emissions trading schemes on the horizon, savings from fuel burn reduction are likely to exceed those from the fuel cost alone. A second cost related opportunity is that, with more advanced thrust management during the departure, airlines might be able to reduce engine wear and associated maintenance costs. Our current simulation however does not model engine wear, so this is still to be confirmed.

Airlines executing noise optimized departures could certainly use this fact in the currently very popular endeavor of convincing their (prospective) customers of their 'greenness' as a company. Similarly, and especially relevant for carriers that are dominant at one of more noise sensitive airports, they can present it to the surrounding communities as one of their efforts of being a good neighbor. For both examples, the airline will see no direct financial savings from operating in an environmentally less harmful way. This could change however, if negative externalities (sometimes called external costs) are priced as well, based on the 'polluter pays principle'. Enforcing this would typically be a governmental responsibility.

\section{B. Government responsibility}

Apart from safety related issues, it is important to realize that government involvement and responsibility with respect to the presented concept is not strictly necessary. It would be limited by providing economic incentives to correct market failure in the management of natural resources and the control of pollution. The term Environmental Fiscal Reform (EFR) is often used in relation to this process ${ }^{9}$.

With respect the custom optimized departure profiles concept, the benefits of applying EFR can probably best be shown using a numerical example, based on the trajectory optimization tool that also forms the basis of this concept. This tool, called NOISHHH, typically has the possibility to significantly reduce the number of people at risk of 
awaking due to fly-over events. Apart from the previously mentioned effects on goodwill and image, there is however no incentive for the airlines to take this into consideration. In fact -compared to a strictly fuel optimized trajectory- an airline even has to accept a slightly increased fuel burn ${ }^{3}$. Comparing the differences in fuel burn with the number potential awakenings, it shows that most avoided awakenings are very inexpensive, with 'exchange rates' sometimes as low as 1000 awakenings to a gallon of jet fuel ${ }^{10}$. This means that the overall societal trade-off should not be very difficult and EFR can be used to help the airline to make the trade-off.

The main government responsibility in this respect is to set the price on environmental performance, for example by applying discounts and surcharges (bonus-malus system). This should direct the airline, not only in making a decision for a particular flight, but also in deciding on investing in technology resulting in less environmental impact. First of all, that applies to the investment decision related to a system as presented here, but could for example also apply to decisions on new equipment.

\section{ANSP responsibility}

The first additional responsibility for the ANSP with respect to the optimal departure profile concept is to define the constraints for the trajectory optimization and to present them to airlines wishing to determine an optimal profile for a certain departure. In the simplest form, these constraints only consist of minimum altitudes with respect to obstacle clearance, together with an altitude restriction on joining the airway at the end of the SID.

Together with these restrictions, it might be convenient to distribute the actual or forecasted meteorological conditions to be used for the profile optimization. Airlines could still decide to actually use this data, or to use similar data from their own sources. Similarly, the ANSPs could be made responsible for providing the airlines with the environmental objectives as determined by the responsible governmental organization. Of course, if pricing mechanisms apply to the environmental impact, this information should be provided to the airlines as well.

When the airline has the optimized profile available, the profile is communicated to the ANSP. The offered trajectory is checked against the (possibly updated) constraints and approved if it meets those constraints. If not, the most basic solution is to simply reject the optimized profile and approve the standard departure procedure. Instead of simply rejecting the offered solution in favor of a standard procedure, more advanced trajectory negotiation is possible as well, although this option is not further explored at this time.

If the profile as requested by the airline is indeed approved by the ANSP, the result is stored for later use. For example, it can be used by the controllers as a reference during the actual flight execution. With two consecutive departures both making use of optimized profiles, it can also be used to calculate the required minimum departure interval, based on four-dimensional (4D) flight execution, with potential positive effects on the runway departure capacity.

\section{Trajectory Optimization}

The actual trajectory optimization method used for testing and illustrating this concept is the NOISHHH-tool, as mentioned earlier. This section gives a short overview of the tool, before discussing the adaptation that were required for optimizing departure profiles instead of departures trajectories.

\section{A. The NOISHHH tool}

To facilitate the design of advanced noise abatement procedures, a tool called NOISHHH is being developed at the Delft University of Technology. The tool can generate routings and flight-paths for both arrivals and departures that minimize the single event environmental impact in the residential communities surrounding the airport, while satisfying all imposed operational and safety constraints. To perform this task, the tool combines a noise model, a dose-response relationship, a geographic information system and a dynamic trajectory optimization algorithm. These different components and optimization methods are discussed here briefly ${ }^{11}$.

The numerical optimization method employed in this study to solve the dynamic trajectory optimization problem is the direct optimization technique of collocation with nonlinear programming (NLP). The collocation method essentially transforms an optimal control problem into a NLP formulation by discretizing the trajectory dynamics. To this end, the time interval of an optimal trajectory solution is divided into a number of subintervals. The individual time points delimiting the subintervals are called nodes. The values of the states and the controls at the nodes are then treated as a set of NLP variables. The system differential equations are discretized and transformed into algebraic equations (implicit integration). The path and control constraints imposed in the original optimal control problem are treated as algebraic inequalities in the NLP formulation.

The flight-path computation is based on a slightly simplified point-mass model, the so-called intermediate model. The underlying assumption for the intermediate model is equilibrium of forces normal to the flight path. 
Because the problem is formulated as a multi-phase trajectory optimization problem, different aerodynamic models can be used for the different parts of a single optimal trajectory. First of all, this property is used to reflect aerodynamic changes because of changes in flap settings. At the same time it is also be used to identify the optimal flap retraction schedule by letting the solver free in choosing the position (or timing) of the phase changes.

To evaluate aircraft flyover noise, a model has been developed that essentially implements the basic methodology employed within the Integrated Noise Model (INM). This model computes the sound exposure levels at specified observer locations. Based on the calculated results, and population density data from the geographic information system the noise performance index is computed using dose-response relationships. For NOISHHH, the most often used one is the noise-awakenings relationship as proposed by the FICAN in $1997^{12}$. This particular relationship provides the percentage of the exposed population expected to be awakened (\%Awakenings) as a function of the exposure to single event noise levels expressed in terms of sound exposure level occurring indoors. An average sound transfer loss of $20.5 \mathrm{~dB}(\mathrm{~A})$ is assumed when calculating the indoor levels based on the outdoor levels as computed by the INM-related method.

The final performance index for this problem is a usually composite function. Depending on the actual application the performance function can be changed, but is typically the weighted sum of the fuel burn and the number of awakenings:

$$
J=\int_{t_{0}}^{t_{f}} \sigma d t+k \cdot A
$$

Where the integral from the initial time $\left(t_{0}\right)$ to the final time $\left(t_{f}\right)$ over the fuel flow $\sigma$ represents the total fuel burn, $A$ is the number of awakenings and $k$ is a user-defined multiplication factor $(k \geq 0)$.

\section{B. Concept Related Changes}

For the optimal departure profiles concept, the lateral part of the flight path is a priori specified and not optimized. Therefore a few adaptations have been made to the original software package. Basically, the point-mass model changes from a three-dimensional to a two-dimensional formulation. This results in the following reductions in the state and control vectors:

$$
\underline{y}:\left(\begin{array}{c}
x \\
y \\
z \\
\chi \\
E
\end{array}\right) \Rightarrow\left(\begin{array}{c}
s \\
z \\
E
\end{array}\right) \quad \underline{u}:\left(\begin{array}{l}
\gamma \\
\mu \\
\eta
\end{array}\right) \Rightarrow\left(\begin{array}{l}
\gamma \\
\eta
\end{array}\right)
$$

With this reduction, the original position coordinates $x$ and $y$ are replaced by (ground plane) distance coordinate $s$. If required (for example for the noise calculations) the actual values of $x$ and $y$ are available for any given $s$ position using a new route definition method. This method determines the actual values by using interpolation on a lookup table. Because the lookup table also includes turn radii, the eliminated bank angle control variable $\mu$ can also be computed for a given s-position, if the corresponding velocity is available.

Although a reduction in state and control variables reduces the problem complexity and therefore leads to reduced computation times, the variable change leads to a more important advantage. In the old situation, a new initial solution had to be generated for each new runway and route combination, because $\mathrm{x}$ and $\mathrm{y}$ coordinates change accordingly. This was a tedious and time-consuming exercise. For the fixed-routing version of the software, this is no longer required. As long as the boundary conditions do not change too radically, any previous solution will typically be accurate enough for the solver to compute the new optimum. This advantage can be exploited to increase levels of automation, if a large number of solutions needs to be generated, as will also be discussed later on.

\section{Other Recent Improvements}

A few other changes have been made to the NOISHHH tool, not related to this specific concept but rather to increase the accuracy of the model. First of all, wind effects were not modeled in previous versions, assuming the True Air Speed (TAS) to be equal to the Ground Speed (GS). Since wind has a significant influence on climb performance, it was added to this version. Please note that the noise propagation modeling itself still does not take wind effects into consideration.

The implementation for this specific problem is not very complicated, because of the assumption that the aircraft will always remain on the prescribed ground track. When looking at the wind triangle as shown in Figure 1, this 
means that the course $\left(\psi_{a}\right)$ is always known for any given position. Together with also known wind speed $\left(V_{w}\right)$, wind direction $\left(\psi_{w}\right)$ and the TAS in the horizontal plane $\left(V_{t} \cos \gamma\right)$, the ground speed $\left(V_{g}\right)$ can be readily solved.

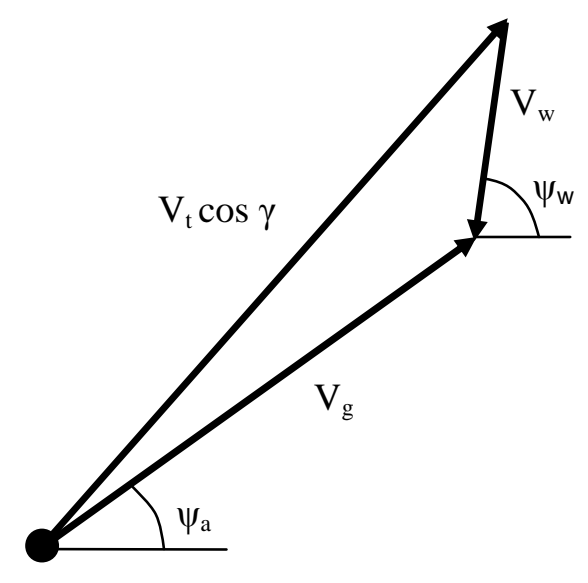

Figure 1: The wind triangle

The wind model itself is at this point not very sophisticated, but rather a list of altitudes with corresponding wind direction and velocity. For the actual altitude, both values are obtained through linear interpolation. Future updates of the wind model itself might be desirable and would not be very complicated.

A second improvement to the previous versions is the availability of calibrated airspeed (CAS). Previously, only the TAS and the Equivalent Air Speed (EAS) were available. EAS was assumed to be equal to Indicated Air Speed (IAS) and was used for the procedure related constraints, like speed constraints at flap changes and general ATC imposed or airframe related speed limits. With the availability of the calibrated speed, it is now the CAS that is assumed to be equal to IAS, which should give a small improvement in the approximation, especially at higher speeds.

Finally, the requirement with respect to the thrust cutback limitation has been adapted. This constraint is used to guarantee sufficient thrust in case of an engine out condition. Previously, commanded thrust was not allowed to be less than $60 \%$ of the maximum available thrust.

$$
0.6 \leq \frac{T}{T_{\max }} \leq 1
$$

This constraint has been reformulated to a performance based constraint that guarantees the ability to proceed with a level and un-accelerated flight in case of complete thrust loss on one engine, awaiting (automatic) thrust restoration on the remaining one(s) to resume the climb. The thrust cutback constraint is therefore now modeled as:

$$
\left(\frac{n-1}{n} T-D\right) \geq 0 \quad \text { and } \quad T \leq T_{\max }
$$

Where $n$ is the number of engines $(n \geq 2), T$ is the commanded thrust and $D$ is the instantaneous drag. This means that the available control authority with respect to the thrust is now a function of aircraft weight, but that speed, flap settings and altitude are also taken into consideration.

\section{Trajectory Synthesis}

Although NOISHHH is suitable to be used in a research environment, it is probably less suitable for an operational environment. One of the main problems stems from the nature of the optimization method. For an arbitrary initial solution, it cannot be guaranteed that the final optimal solution can always be reached. And even if the solver will reach a converged solution, it cannot be guaranteed it will do so within a specified amount of time. Therefore the presented tool itself cannot be relied upon in an operational environment.

A possible solution for the problem is to create a database of optimal profiles, such that a suitable profile for a specific flight is always available when required. However, there are a lot of parameters involved in the profile optimization problem. Some are discrete and lead to a limited (or at least controlled) number of combinations like aircraft type, runway and departure route to be used. Others are continuous over a certain interval, like temperature and take-off weight. These intervals need to be discretized when using this 'database-of-solutions' approach. This leads to a trade-off regarding interval spacing with respect to these continuous parameters. Using small intervals allows for a better approximation of the actual conditions, but results in the unworkable situation of a practically infinite number of profiles that needs to be in the database. Using wide intervals is good for the database size, but can result in a poor approximation. Although using interpolation in the n-dimensional space of the continuous parameters may help in improving this, there is still a certain risk present. Synthesizing a trajectory from a number of other trajectories does not automatically guarantee that the result lies within the performance capabilities of the aircraft.

Therefore, the approach proposed here is to construct a control vector by means of interpolation from the solutions database instead of an interpolated state vector. With use of the obtained control vector and the same 
models as used by NOISHHH the resulting state vector (the actual profile or trajectory) can simply be computed almost instantly while using the actual parameters instead of the approximated ones. This can than be done using the same performance and noise models as the optimization software itself, and without the risk of generating a profile that is outside the capabilities of the aircraft. This process is illustrated in Figure 2.

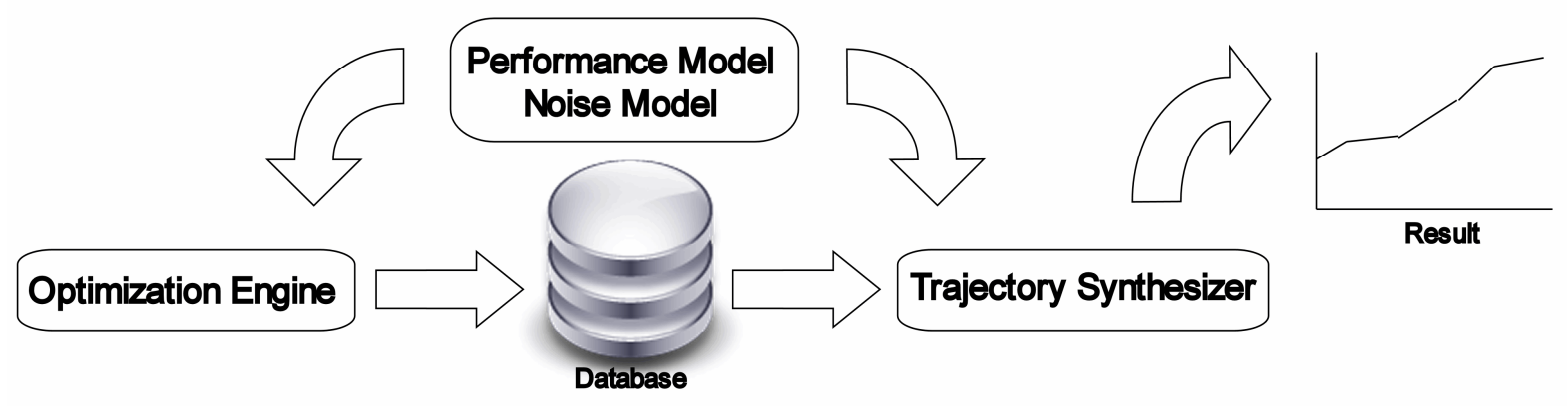

Figure 2: The process of optimal trajectory synthesis

As a side-note, it is important to realize that the reliability, stability and computing effort may differ among different optimization methods, solvers, tools and computing hardware. This means that the need for an alternative solution generation method as proposed here may also be dependent on the chosen implementation. Another important factor is this respect is the requirements of the (in-time) availability of optimal solutions for all fights. In other words, for what percentage of the flights an operator would be satisfied with for example using a standard departure procedure as backup in case the optimal profile is not available or late.

Similarly, allowing for the use of sub-optimal solutions might be an option, where sub-optimal solutions are solutions that show improved performance compared to standard procedures but are not guaranteed to be optimal. It might be possible to develop a relatively stable tool that is able to generate such sub-optimal solutions within a predetermined amount of time.

\section{Numerical Example}

\section{A. Procedures, Aircraft and Routing}

The optimized profiles used for this numerical example are based on the description as provided by an FAA advisory circular ${ }^{13}$. Based on the description for the so called close-in community NADPs, these are the most important constraints for the optimization exercise:

- Flap (and slats) retraction should be commenced before thrust cutback is initiated.

- Thrust cutback should not take place before reaching an altitude of $800 \mathrm{ft}$.

- The thrust cutback restriction as discussed in section III is enforced as long as the aircraft is not in clean configuration.

- Flap retraction (and therefore also thrust cutback) is not allowed before reaching an altitude of $500 \mathrm{ft}$. (not mentioned in the advisory circular, but added for reasons of safety)

The reference procedure - which is to be compared to the optimized profile - is the ICAO-A departure. When using this procedure the aircraft performs the initial climb at $\mathrm{V}_{2}$ plus 10 to 20 knots. At $1500 \mathrm{ft}$, the thrust setting is reduced from take-off thrust to climb-thrust, while maintaining the same indicated airspeed. At $3000 \mathrm{ft}$ and still at climb thrust, the aircraft is accelerated and the transition towards the clean configuration is initiated.

To enable a realistic comparison of both procedures, the ICAO-A departure is also optimized using NOISHHH. However, the definition of the procedure eliminates most optimization opportunities. Basically, NOISHHH will only determine the fuel optimal trade-off between a steeper climb and a quicker acceleration after reaching $3000 \mathrm{ft}$. Both departures will be calculated with the wind model disabled (TAS equal to GS), and are based on the ISA standard atmosphere conditions with respect to temperature, pressure and density for a given altitude.

The aircraft model selected for this example belongs to the twin engine Boeing 737-300 airliner, one of the four available type models currently implemented in NOISHHH. The take-off mass used for this simulation is $54386 \mathrm{~kg}$ (119900 lbs), which corresponds to one of the intermediate departure weight as defined by INM. It is assumed that the weight of the aircraft is constant throughout the departure phase.

American Institute of Aeronautics and Astronautics 092407 
Both the optimized departure as well as the standard departure involve the VALKO SID from Amsterdam's runway 24. This route is for example used for departures with destination London. The constraints for the last point of the optimized trajectories are chosen such that the location coincides with the location of VALKO. For a comparison as accurate as possible, the required energy state at the endpoint is provided as well. All results should end in VALKO at $6000 \mathrm{ft}$ (based on the default FL 60 crossing condition and assuming ISA standard atmospheric conditions), at 250 knots CAS.

Since the modeling does not include the take-off roll, the initial conditions had to be set as well (instead of all simply being zero). The initial conditions that where chosen are those that correspond to the situation where the aircraft reaches an altitude of $50 \mathrm{ft}$, directly after take-off. INM flight path computation data has been used to obtain the corresponding remaining state variables.

\section{B. Results}

As mentioned before, an ICAO-A departure is compared to the optimized departure. However, because of the variable trade-off between fuel and awakenings, two different optimized results will be shown. The first optimized result is fuel optimal only, which corresponds to a k-value of zero in eq. 1 . The second optimized result is a combined fuel and awakenings optimization.

Multiple results have been generated for different values of $\mathrm{k}$, ranging from solutions that hardly differ from the fuel optimal solution, to somewhat more exotic results completely dominated by noise considerations. The composite result shown is the one that belongs to a $\mathrm{k}$-value of $0.025 \mathrm{~kg}$ fuel per awakening. This value translates in a trade-off of 40 awakenings per $\mathrm{kg}$ of fuel, which means that the solver can move the solution towards a more noise optimal solution, as long as the reduction in the number of awaking is at least 40 for every kilogram of additional fuel burn.

Figure 3 shows the altitude profiles for all three departures, where the altitude is plotted versus the distance. When looking at these three profiles, it is clear that both optimized departures show a less steep initial climb. The fuel-optimized departure however, catches up with the ICAO-A departure, and reaches the final altitude in the least distance. The fuel and awakenings combined profile clearly climbs on reduced less engine power and even has a short level segment in order to reduce the thrust as much as possible at a particular geographic location.

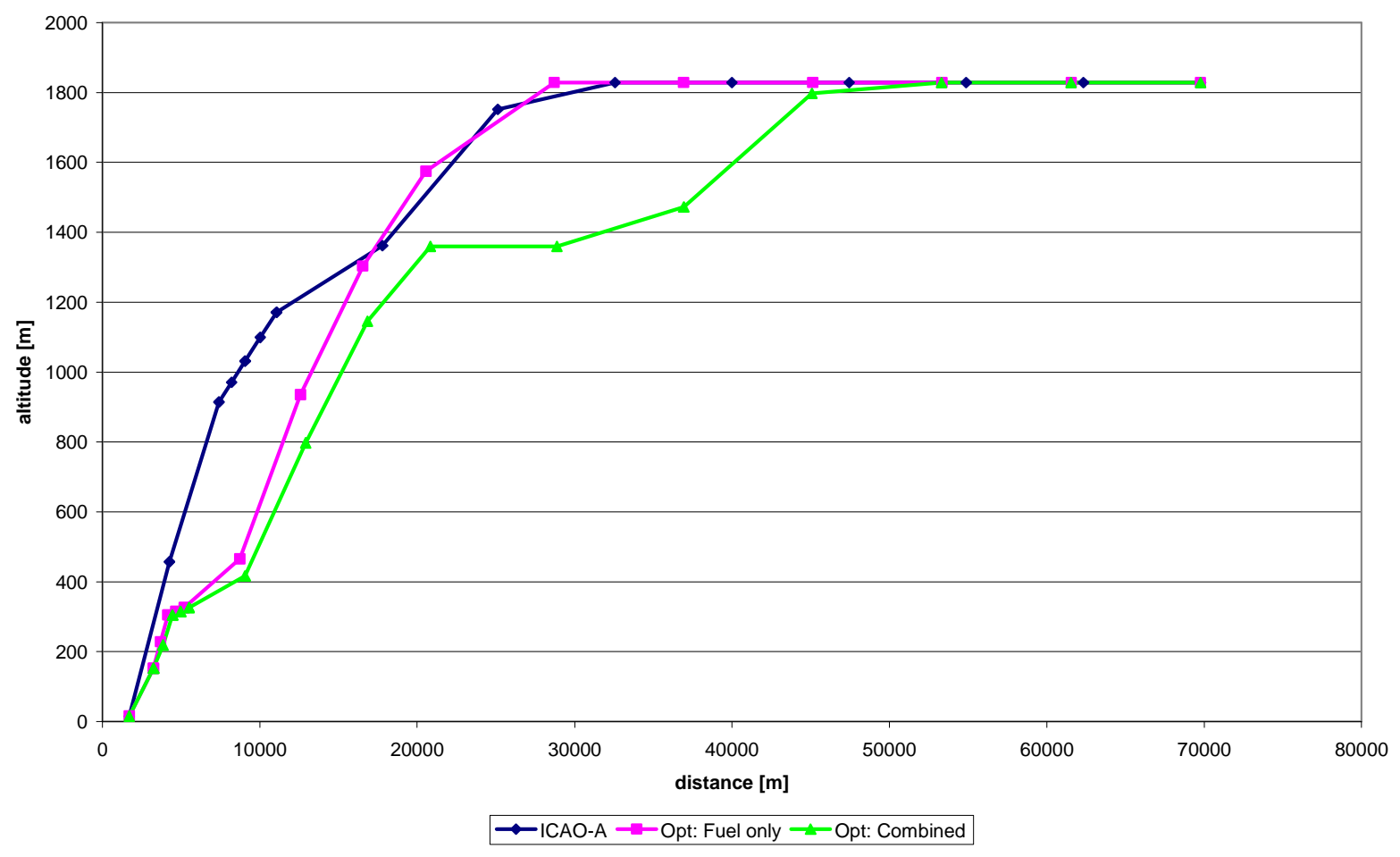

Figure 3: altitude profile for the different departure procedures 
Figure 4 shows the speed profiles of all three departures, plotted versus the time. Both optimized departures have similar speed profiles, clearly different from the one corresponding to the ICAO-A departure. Both optimized profiles show acceleration early in the departure, allowing for an early flap retraction. The ICAO-A departure on the other hand maintains a constant IAS, resulting in a slightly increasing TAS until reaching $3000 \mathrm{ft}$. The differences in speed automatically lead to another difference between the profiles. The ICAO-A departure reaches the final point considerably later than the other two.

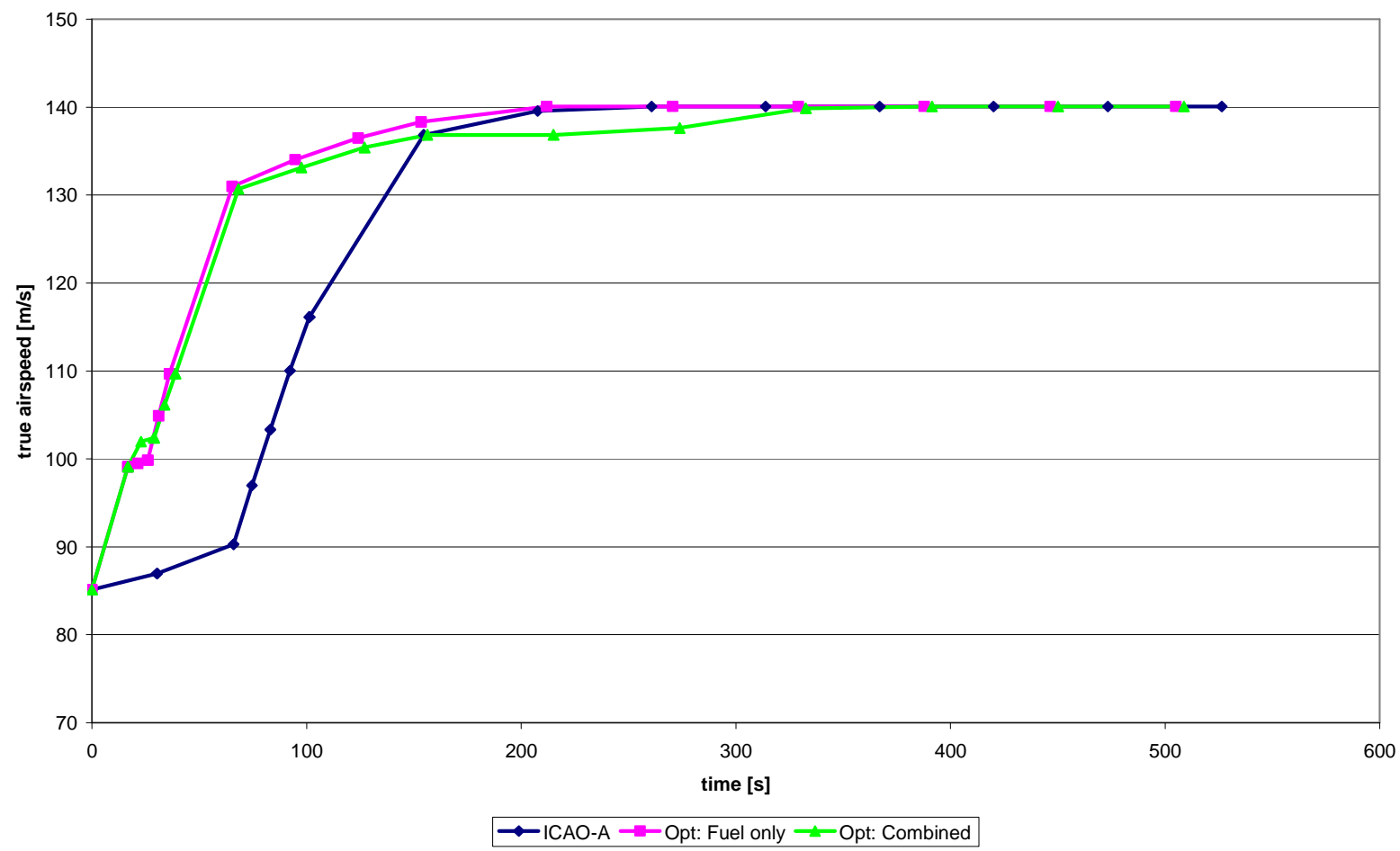

Figure 4: speed profiles for the different departure procedures

Figure 5, Figure 6 and Figure 7 show the noise load for the three departures (Sound Exposure Level, SEL). 


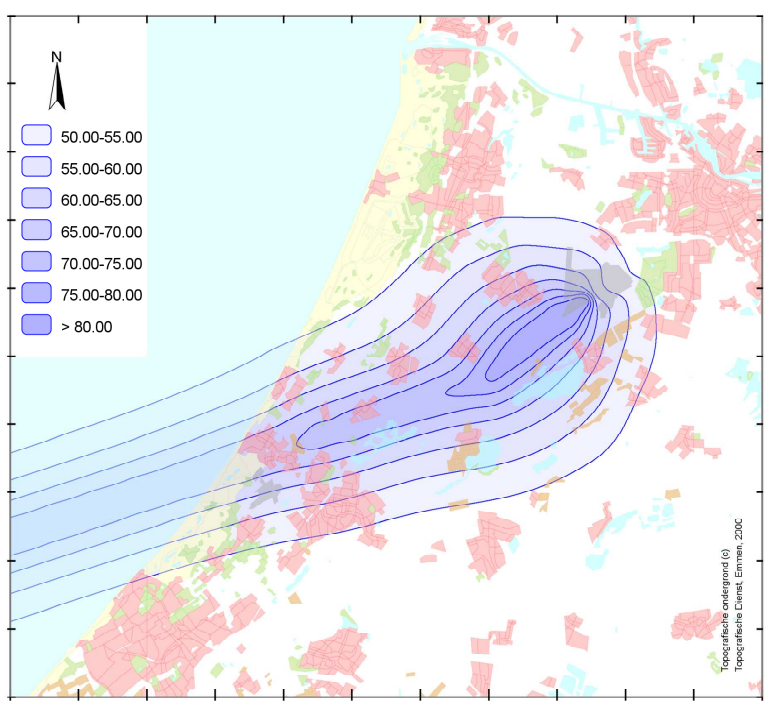

Figure 5: ICAO-A departure, sound exposure level in $\mathrm{dB}(\mathrm{A})$

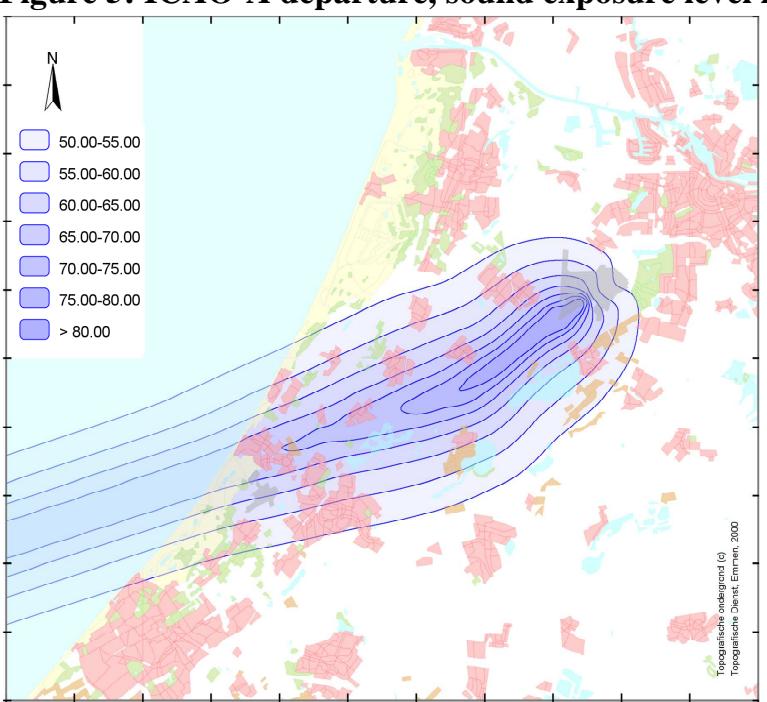

Figure 6: Fuel optimized departure, sound exposure level in $\mathrm{dB}(\mathrm{A})$

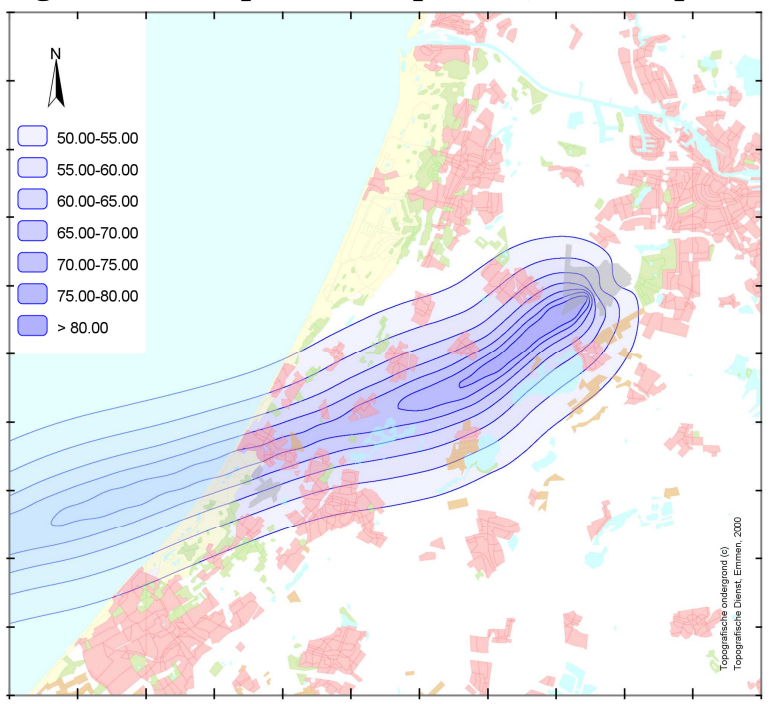

Figure 7: Fuel and noise combined optimized departure, sound exposure level in $\mathrm{dB}(\mathrm{A})$

10

American Institute of Aeronautics and Astronautics 092407 
Finally, Table 1 compares the three departures on total fuel burn (excluding the take-off roll), the number of expected awakenings and time that is required to reach the final point. Both optimized procedures use less fuel, result in fewer awakenings and arrive at the final point about twenty seconds earlier. The differences between the two optimized procedures are less distinct. For the combined solution, almost three hundred fewer awakenings can be achieved (an additional 9 percent), at the cost of $3 \mathrm{~kg}$ of fuel and 3 seconds or flying time.

Table 1: Comparison of results

\begin{tabular}{|l|c|c|c|}
\hline Result type & Fuel burn $[\mathbf{k g}]$ & Awakenings & Time [s] \\
\hline ICAO-A & 510 & 3322 & 526 \\
\hline Optim. Fuel only & $482(-5.5 \%)$ & $2808(-15.5 \%)$ & $505(-4.0 \%)$ \\
\hline Optim. k=0.025 & $485(-4.9 \%)$ & $2516(-24.3 \%)$ & $509(-3.2 \%)$ \\
\hline
\end{tabular}

\section{Conclusion}

A concept has been developed for advanced noise abatement departure procedures, based on custom optimized departure profiles. In this concept, an airline generates an optimized departure profile for a specific flight, based on constraints and objectives as set by different stakeholders. After receiving approval from ATC concerning the desired trajectory, the concerning flight can depart using the optimized profile, instead of a general procedure.

The software that has been developed for generating the optimized profiles is based on a previously developed trajectory optimization tool. Although no longer authorized to optimize in the horizontal plane, it still shows promising results for a test case, when compared to a standard procedure. Based on the examined performance indicators, the optimized profiles clearly outperform the standard ICAO-A procedure. As such, they have the potential to both reduce fuel burn as well as noise exposure. At the same time, no negative effects on capacity are expected, although computations or simulations have not yet been performed to confirm this.

The optimized profiles are primarily based on aircraft capabilities and avionics requirements have not been assessed at this time. Future research will need to be conducted with respect to this area. At the same time, experiments will have to proof whether it is indeed possible to create a feasible detailed operational concept based on the custom optimized profiles idea.

\section{References}

${ }^{1}$ Erkelens, L.J.J., "Research into New Noise Abatement Procedures for the $21^{\text {st }}$ Century", AIAA Guidance, Navigation and Control Conference, AIAA, Reston, VA, 2000.

${ }^{2}$ Davison Reynolds, H. J., Reynolds, T. G., and Hansman, R. J., "Human Factors Implications of Continuous Descent Approach Procedures for Noise Abatement", Air Traffic Control Quarterly, Vol 14, No. 1, pp. 25-45, 2006.

${ }^{3}$ Visser, H.G., and Wijnen, R.A.A., "Optimization of Noise Abatement Arrival Trajectories", AIAA Guidance, Navigation, and Control Conference and Exhibit, AIAA, Reston, VA, 2001

${ }^{4}$ Visser, H.G., and Wijnen, R.A.A., "Optimization of Noise Abatement Departure Trajectories", Journal of Aircraft, Vol. 38, No. 4, July-August 2001.

${ }^{5}$ Visser, H.G., "Environmentally-Optimized Air Traffic Conflict Resolutions", AIAA Aviation Technology, Integration and Operations Conference, AIAA, Reston, VA, 2007

${ }^{6}$ Prats Menéndez, X., Nejjari, F., Puig Cayuela, V., Quevedo Casin, J.J., Mora Camino, F., “A Framework for RNAV trajectory generation minimizing noise nuisances", Second International Conference on Research in Air Transportation, Faculty of Transport and Traffic Engineering, Belgrade, Serbia, 2006.

${ }^{7}$ Vormer, F.J., Mulder, M., van Paassen, M.M., and Mulder, J.A., "Optimization of Flexible Approach Trajectories Using a Genetic Algorithm", Journal of Aircraft, Vol 43, No. 4, July-August 2006.

${ }^{8}$ SESAR Consortium, “The ATM Target Concept - D3”, EUROCONTROL, Brussels, Belgium, 2007

${ }^{9}$ Ekins, P., Speck, S., "Proposals of environmental fiscal reforms and the obstacles to their implementation", Journal of Environmental Policy and Planning, Vol. 2, No. 2, 2000.

${ }^{10}$ Hogenhuis, R.H., Hebly, S.J., Visser, H.G., "Optimization of RNAV Noise Abatement Arrival Trajectories", 26 ${ }^{\text {th }}$ International Congress of the Aeronautical Sciences, ICAS, Amsterdam, The Netherlands, 2008

${ }^{11}$ Visser, H.G., Hebly, S.J., Wijnen, R.A.A., "Improving the Management of the Environmental Impact of Airport Operations", in Gustavsson, F.N., New Transportation Research Progress, Nova Science Publishers, Hauppage, NY, 2008

${ }^{12}$ Federal Interagency Committee on Aviation Noise, "Sleep Disturbance caused by Aviation Noise," 1997

${ }^{13}$ Federal Aviation Administration, "Noise Abatement Departure Procedures", AC 91-53A, 1993 